\title{
Unusually Large Exponent for the Inverse Power-Law Blinking of Single Chromophores
}

\author{
Der-Hau Lee and Jau Tang* \\ Research Center for Applied Sciences, Academia Sinica, Taipei 115, Taiwan, and Department of Photonics, \\ National Chiao-Tung University, Hsinchu, Taiwan
}

Received: February 29, 2008; Revised Manuscript Received: July 02, 2008

In this study of single-particle fluorescence intermittency, we investigate some interesting blinking phenomena of chromophores by Yeow et al. (J. Phys. Chem. A 2006, 110, 1726), showing behavior very different from the usual quantum dots. Using Monte Carlo simulations we demonstrate that the short-time blinking statistics are generally nonexponential but could be characterized by an inverse power law with an effective exponent $m$ far exceeding 2, as observed in some single-chromophores experiments. We show that $m$ for the on-events decreases with the effective diffusion correlation time which increases with light intensity. This work offers an explanation to why $m$ could change from 2.8 to 2.0 in their experiments as the light intensity is quadrupled.

\section{Introduction}

The size-dependent photophysics of quantum dots (QDs) has generated broad research interest in recent years with many potential applications. ${ }^{1-3}$ Furthermore, the development of single-molecule techniques also speeds up the investigation of unusual properties of nanoparticles, single organic chromophores, and biomolecules. One of the most intriguing features in single-molecule or single-particle spectroscopy is fluorescence intermittency or commonly called blinking. Each single nanoparticle or molecule under continual light illumination displays a histogram with randomly distributed light and dark periods. The waiting time distribution for either "on" or "off" events is often characterized by an inverse power law. Such a phenomenon has been under intensive investigation experientially ${ }^{3-9}$ and theoretically. ${ }^{3,4,10-15}$ There were numerous physical models proposed to rationalize such behavior; for example, Bawendi and his co-workers presented a dynamic model of tunneling between core and charged states, ${ }^{4}$ and Kuno et al. suggested several a few kinetic models of fluorescence intermittency. ${ }^{5}$ Verberk et al. $^{6}$ related the exponent to the barrier width. Recently, one of the authors (J.T.) proposed with Marcus a diffusion-controlled electron transfer (DCET) mechanism to account for the power-law blinking phenomenon. ${ }^{14}$ This model involves a diffusion-controlled charge transfer process in energy or configuration space. For normal diffusion, that model leads to an exponent $m$ at $3 / 2$ for the inverse power law. For anomalous cases, the exponent could lie between 1 and 2 . The exponent is also predicted to be less than 2 according to the other model of Verberk et al. ${ }^{6}$ Although it is true that most of the observed exponents in single-particle experiments for various systems are smaller than 2, a few violations do occur. For example, according to Cichos et al., ${ }^{7} m=2.2 \pm 0.1$ in $\mathrm{Si}$ nanocrystals, and in the single-chromophore study by Yeow et al. ${ }^{16} \mathrm{~m}$ can vary from 2.8 to 2.0 , depending on light intensity. Although one could argue that, given the uncertainty in $m$, the Si case might not be a serious violation of the existing models, the experimental observation of $m=2.8$ would certainly raise concern about our general understanding of the underlying mechanism for blinking phenomena.

\footnotetext{
* Corresponding author. E-mail: jautang@gate.sinica.edu.tw.
}

According to our more recent DCR (diffusion-controlled reaction) model ${ }^{15}$ involving a 2 -D potential with both fast and slow reaction coordinates $(q$ and $Q)$, we have shown that after the reduction of the fast coordinate one obtains an equivalent 1-D DCR model with the reaction rate at the sink characterized by a Gaussian dependence on the slow coordinate $Q$ and its second moment depends on the free energies and the force constants of the 2-D paraboloidal potentials. The major difference of this reduced 1-D DCR model from the usual DCET model is in that the sink at the energy level crossing between the light and the dark states is no longer a Dirac $\delta$-function but a Gaussian with a finite spread. In this study, we will elaborate on this more general DCR model, using Monte-Carlo simulations to demonstrate some interesting features and to compare model predictions with available experimental data. We shall show that the exponent of the inverse power law depends on the details of the diffusion-controlled reaction mechanism, such as the root-mean-square of the fluctuations of the transition rate and the diffusion correlation time which could be influenced by changes in light intensity. This treatment is an extension of the model of Sumi and Marcus ${ }^{17}$ which was intended for electron transfers of an ensemble system, but here we consider singleparticle blinking. As explained earlier, ${ }^{12}$ in dealing with singleparticle blinking, one should use a decoupled rate equation as we do here.

2. Reduced 1-D DCR model. According to the 2-D DCR model $^{15}$ as illustrated in Figure 1, one consider charge transfer between the light and dark states involving two paraboloidal potentials $U_{1}(X, Y)=\kappa_{x} X^{2} / 2+\kappa_{y} Y^{2} / 2$ and $U_{2}(X, Y)=\kappa_{x}(X-$ $\left.X_{0}\right)^{2} / 2+\kappa_{y}\left(Y-Y_{0}\right)^{2} / 2+\Delta G^{0}$, with a force constant $\kappa_{x}$ and $\kappa_{y}$, respectively. By reduction of the fast reaction coordinate, one obtains a reduced 1-D diffusion-controlled reaction rate equation that involves 1-D diffusion and a Gaussian sink term describing the escape from a light state to a dark state and vice versa. For the "on"-events one has ${ }^{15}$

$$
\frac{\partial}{\partial t} \rho_{1}(Q, t)=\frac{1}{\kappa_{y} \beta \tau_{c}}\left(\frac{\partial^{2}}{\partial Q^{2}}+\kappa_{y} \beta \frac{\partial}{\partial Q} Q\right) \rho_{1}(Q, t)-k_{1}(Q) \rho_{1}(Q, t)
$$

The effective diffusion constant is given by $1 / \kappa_{y} \beta \tau_{c}$ and the fluctuating forward transition rate is given by 
$k_{1}(Q)=k_{0} \exp \left(-\beta \Delta G_{1}(Q)\right)=$

$k_{0} \exp \left[-\beta \kappa_{\text {eff }}\left(Q-Q_{\mathrm{a}}\right)^{2} / 2\right]$

$$
k_{0} \equiv \frac{2 \pi\left|V_{\mathrm{ex}}\right|^{2}}{\hbar} \frac{1}{\sqrt{4 \pi \lambda_{x} k_{\mathrm{B}} T}}
$$

where the activation energy $\Delta G_{1}(Q)=\kappa_{\mathrm{eff}}\left(Q-Q_{\mathrm{a}}\right)^{2} / 2$, $\kappa_{\text {eff }} \equiv \kappa_{y} \lambda_{y} / \lambda_{x}$ and $Q_{\mathrm{a}}=\left(\lambda_{x}+\lambda_{y}+\Delta G^{0}\right) / \sqrt{2 \lambda_{y} \kappa_{y}}$. The initial condition for an "on"-event is $\rho_{1}(Q, 0)=\exp \left(-\beta \kappa_{\text {eff }}\left(Q-Q_{\mathrm{a}}\right)^{2 /}\right.$ $2) / \sqrt{2 \pi / \beta \kappa_{\text {eff }}}$. For the "off"-events one has

$$
\begin{aligned}
& \frac{\partial}{\partial t} \rho_{2}(Q, t)= \\
& \frac{1}{\kappa_{y} \beta \tau_{c}}\left(\frac{\partial^{2}}{\partial Q^{2}}+\kappa_{y} \beta \frac{\partial}{\partial Q}\left(Q-Y_{0}\right)\right) \rho_{2}(Q, t)-k_{2}(Q) \rho_{2}(Q, t)
\end{aligned}
$$

where the reverse escape rate

$$
k_{2}(Q)=k_{0} \exp \left(-\beta \Delta G_{2}(Q)\right)=k_{0} \exp \left[-\beta \kappa_{\text {eff }}\left(Q-Q_{\mathrm{b}}\right)^{2} / 2\right]
$$

The activation energy for the reverse ET is defined as $\Delta G_{2}(Q)$ $=\kappa_{\text {eff }}\left(Q-Q_{\mathrm{b}}\right)^{2} / 2$, where $Q_{\mathrm{b}}=\left(-\lambda_{x}+\lambda_{y}+\Delta G^{0}\right) / \sqrt{2 \lambda_{x} k_{y}}$. Equations 1and 2 indicate that the escape rate fluctuates in time via a stochastic variable $Q$ because the activation energy fluctuates in time with a quadratic dependence on $Q$. The fluctuations in $Q$ can be characterized by a Gaussian process which can be described mathematically as a 1-D diffusion equation. Equations 1 and 2 essentially describe the population evolution of a single-particle system with a fluctuating escape rate, where the fluctuations of the stochastic variable $Q$ could be characterized by a Gaussian process.

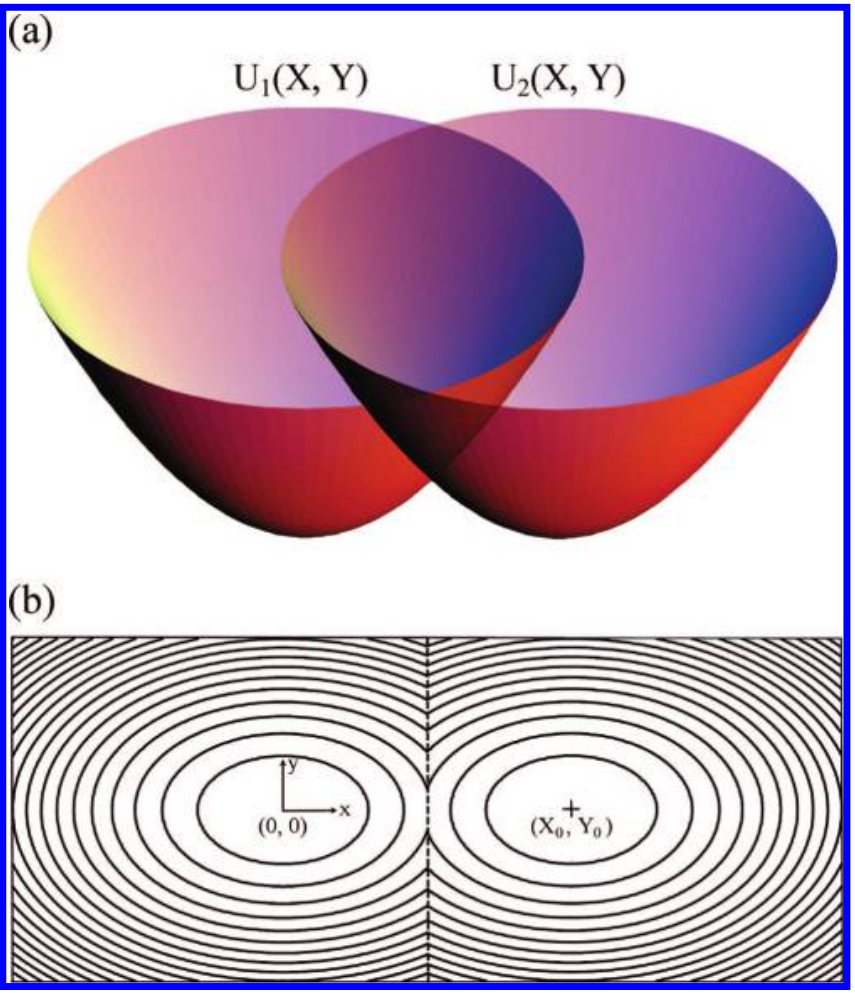

Figure 1. (a) Surface plot of two paraboloidal potentials for the photoexcited neutral light state and the charge-separated dark state in a 2-D reaction coordinate with a fast coordinate $X$ and a slow reaction coordinate $Y$. (b) Contour plot of the 2-D potentials.
In the presence of continual light illumination and fast fluorescence decay from the photoexcited "light" state $\mid 1>$ to the ground state $10>$, a fast population quasi-equilibrium is established between the light state and the ground state. Under continual light illumination at a pumping rate $P$ and a fluorescence decay rate $\gamma$ from $\mid 1>$ to $10>$, eq 1 needs to be modified as

$$
\begin{aligned}
& \frac{\partial}{\partial t} \rho_{0}(Q, t)=\gamma \rho_{1}(Q, t)-P \rho_{0}(Q, t) \\
& \frac{\partial}{\partial t} \rho_{1}(Q, t)=P \rho_{0}(Q, t)-\gamma \rho_{1}(Q, t) \\
&+\frac{1}{\kappa_{y} \beta \tau_{\mathrm{c}}}\left(\frac{\partial^{2}}{\partial Q^{2}}+\kappa_{y} \beta \frac{\partial}{\partial Q} Q\right) \rho_{1}(Q, t)-k_{1}(Q) \rho_{1}(Q, t)
\end{aligned}
$$

Because the blinking usually involves a slow time scale on the order of sub-microseconds or longer, the photoexcitation of the ground state $\mid 0>$ to $\mid 1>$ and the rapid fluorescence decay from $\mid 1>$ to $\mid 0>$ causes a very fast population recycling between $\mid 1>$ and $\mid 0>$. After the initial induction period, one can simplify the above coupled rate equations to become a reduced rate equation for the population in $11>$ as

$$
\begin{aligned}
& \frac{\partial}{\partial t} \rho_{1}(Q, t)= \frac{1}{\kappa_{y} \beta \tau_{\mathrm{c}}}\left(\frac{\partial^{2}}{\partial Q^{2}}+\kappa_{y} \beta \frac{\partial}{\partial Q} Q\right) \rho_{1}(Q, t) \\
& \quad-k_{0} \exp \left[-\beta \kappa_{\text {eff }}\left(Q-Q_{\mathrm{a}}\right)^{2} / 2\right] \rho_{1}(Q, t) \\
& \tau \equiv \tau_{\mathrm{c}}(P+\gamma) / P
\end{aligned}
$$

Equation 4 is similar to eq $1 \mathrm{a}$, and it represents a diffusioncontrolled transition with a $Q$-dependent escape rate, except that one now needs to use an effective diffusion correlation time $\tau$. This effective correlation time constant becomes longer at a smaller light intensity (linearly related to the pumping rate $P$ ) because the effective time spent in the ground-state is longer which does not contribute to diffusion.

According to this model, we have assumed 2-D potentials as shown in Figure 1 describe electron transfer between the photoexcited light state and the charged dark state. Because of a large time-scale separation between the fast and the slow reaction coordinates, the reduction of the fast reaction coordinate in this 2-D reaction coordinate greatly simplifies the diffusioncontrolled reaction model to become 1-D model but with a sink which might have a finite width instead of the usual Dirac's $\delta$-function at the energy-level crossing. In addition, the reduction from eq 3 to eq 4 indicates that the diffusion for the photoexcited light state is light-intensity dependent because the effective diffusion correlation time $\tau$ depends on the pumping rate $P$. Temperature effects on diffusion can enter this dynamics via its influence on $\tau_{\mathrm{c}}$. In contrast, the dark state is insensitive to changes in light intensity. ${ }^{14}$

3. Simulation Results and Discussion. Because eq 4 cannot be solved analytically, we use Monte Carlo approach to simulate 1-D random walk with a Gaussian-shaped population sink. Here we first describe how the simulations were done. To simulate 1-D diffusion we consider random hopping along a 1-D discrete lattice point array with a unit time per hopping trial $1 \mathrm{~ms}$. We set a threshold parameter $\xi$ as a criterion to decide whether a particle should hop either to the right, to the left or to remain at the same lattice point. For each hopping event, a random number $x$ between 0 and 1 is generated. If $x<\xi$ the particle is allowed to hop to the right, and if $x<1-\xi$ the particle hops to the left; otherwise its position remains unchanged. The calculated probability for a particle to remain at the origin in a 


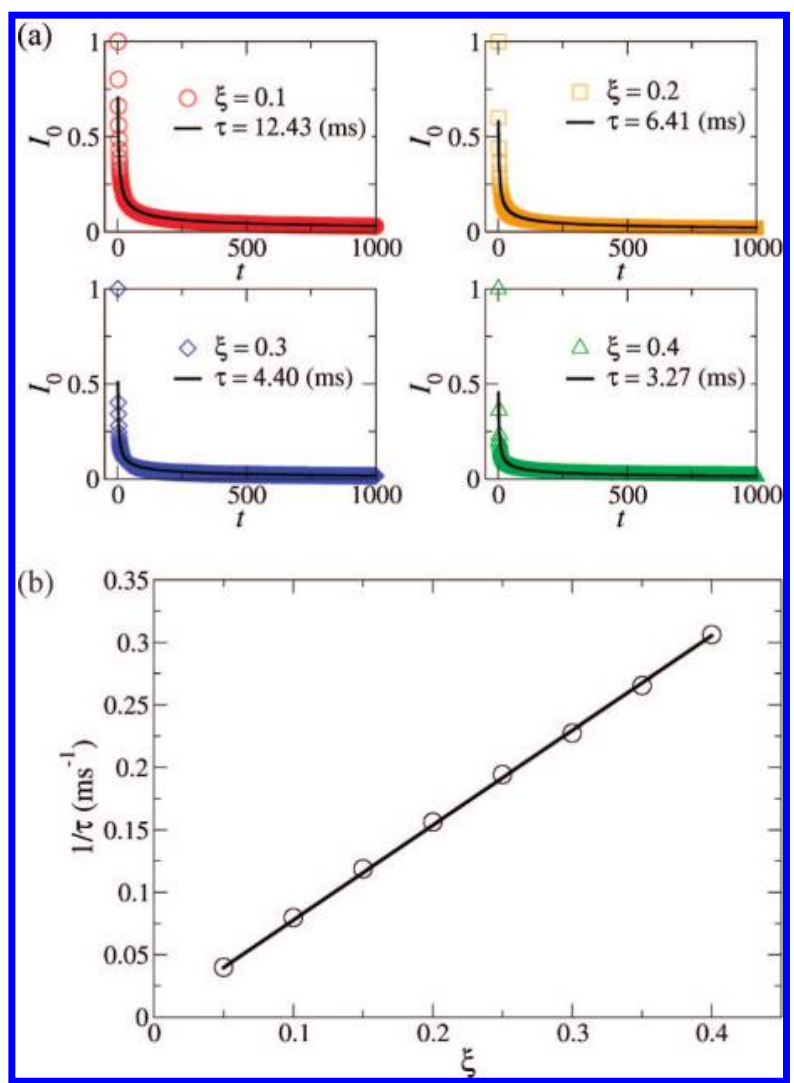

Figure 2. (a) The calculated probability $I_{0}$ at time $t$ for a particle to remain at the origin in a 1-D discrete random walk process. The fitted curve and value for $1 / \tau$ are based on eq 5 . In the simulation, we used threshold parameter $\xi=0.1-0.4$, a time step of $1 \mathrm{~ms}$ for each discrete hopping along a 1-D lattice point array with a total of $9.5 \times 10^{5}$ events. (b) The linear relationship between $1 / \tau$ and the threshold parameter $\xi$. From such dependence one can determine the effective diffusion correlation time constant $\tau$ for a given $\xi$.

pure 1-D discrete random walk process without a sink is shown in Figure 2a. In the curve fitting of Figure 2a we use the following normalized surviving probability

$$
I_{0}(t)=\frac{1}{\sqrt{1+4 \pi \Delta^{2} t / \tau}}
$$

At time zero, $I_{0}(0)=1$ and becomes the well-known $I_{0}(t) \approx$ $1 / \sqrt{4 \pi D t}$ at longer times, where the diffusion constant $D$ is defined as $D=\Delta^{2} / \tau$. Figure $2 \mathrm{~b}$ shows a linear relationship between $\xi$ and $1 / \tau$ and from which we can extract the diffusion correlation time $\tau$ for a given $\xi$.

To simulate blinking phenomena and to determine blinking statistics which is defined as the waiting time distribution of either "on" or "off" period, we extend the above simulation procedure for discrete hopping along a 1-D chain and include a Gaussian-shaped population sink. We define an escape rate $W_{12}$ from the sink and a root-mean-square $\sigma$ for the Gaussian sink. To obtain blinking statistics with a reliable statistics, a very long histogram for the single-particle blinking was generated, typically with $10^{6}$ to $10^{7}$ events. We usually simulate such histograms with about one million on-off events. The log-log plot of $P_{\text {on }}(t)$ in the case of a Dirac $\delta$-function sink with high escape rates $\left(W_{12}=0.62,0.60\right.$, and $\left.0.58 \mathrm{~ms}^{-1}\right)$ were given in Figure $3 \mathrm{a}$, indicating an exponent of $3 / 2$ as expected from the previous DCET model. With an increase of the sink width, as illustrated in Figure 3b, the short-time exponent can become much larger than $3 / 2$ although the long-time behavior is similar
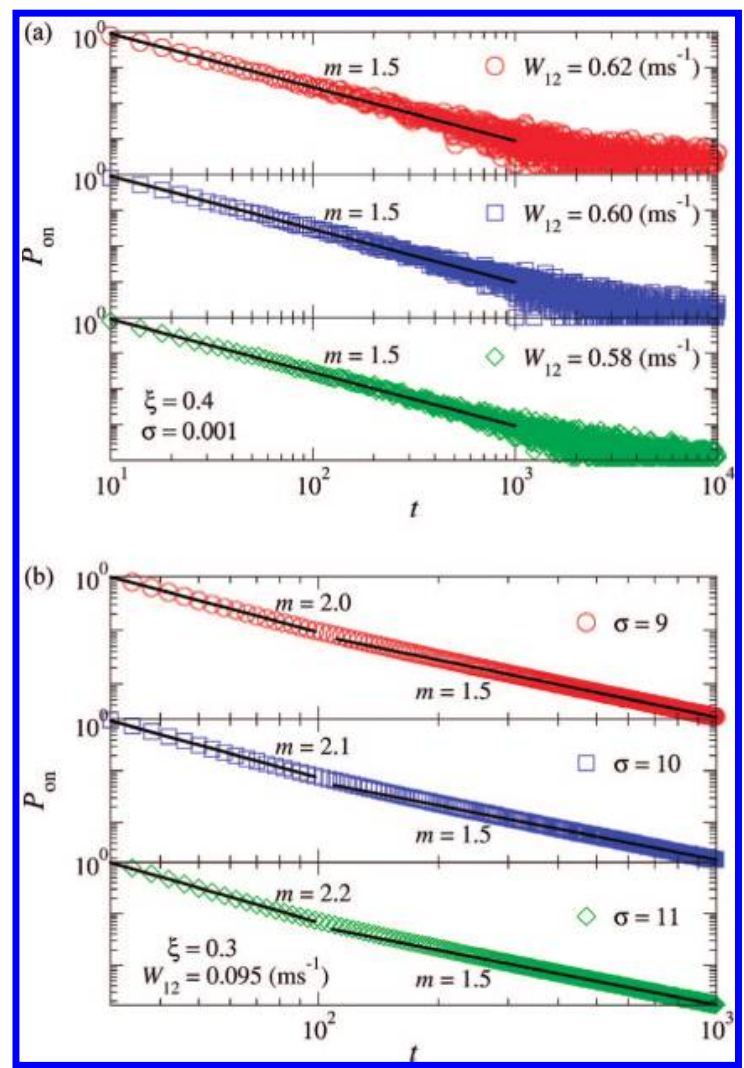

Figure 3. (a) $\log -\log$ plot of the normalized on-time blinking statistics $P_{\text {on }}(t)$ at $W_{12}=0.62,0.60$, and $0.58 \mathrm{~ms}^{-1}$, respectively. A very narrow sink with $\sigma=0.001$ (close to a Dirac $\delta$-function) was assumed. The curves indicate an inverse power law with an exponent very close to 1.5. In the simulations we used $10^{6}$ events, time unit $=1 \mathrm{~ms}$, bin size $=4 \mathrm{~ms}$, and $\xi=0.4$. (b) $\log -\log$ plot of the normalized on-time blinking statistics $P_{\text {on }}(t)$ at $\sigma=9,10$, and 11, respectively. In all cases with $9.5 \times 10^{7}$ events, $W_{12}=0.095 \mathrm{~ms}^{-1}, \xi=0.3$, time step $=1 \mathrm{~ms}$, bin size $=4 \mathrm{~ms}$ were used. The increase of the sink width causes the short-time exponent to increase beyond 2 in these examples, although the long-time exponent remains at $3 / 2$ as usual.

to the result of the DCET model. Now we illustrate the influence of a Gaussian-shaped sink for power-law decay by Figure $4 a-c$. It is shown that bending of the slope takes place at short times if escape rate is smaller. The curves exhibit unusually large slope at very short times. The slope decreases and approaches $3 / 2$ at longer times. Thus, our model simulations at longer times shown here reproduce the feature of normal diffusion described previously. ${ }^{12}$ In addition, our model also allows the existence of a usually large exponent if the population sink has a broad width and the escape rate is faster than the 1-D diffusion rate.

To demonstrate the model applications, here we compare the experimental data of single-chromophores measurements at three light intensities ${ }^{16}$ with our model prediction. In Figure 5 we show the calculated exponent for the inverse power-law blinking as a function of the inverse diffusion correlation time $1 / \tau$ at several parameter settings for $\xi$ and $W_{12}$. According to Yeow et al., ${ }^{16}$ when the light intensity is increased by two and four times, the exponent $m$ changes from 2.8 to 2.4 and then to 2.0. We can reproduce such dependence not only qualitatively but also quantitatively. Because the diffusion in the energy space is photoinduced, the diffusion becomes faster, or the diffusion correlation time $\tau$ becomes shorter, at a higher light intensity (or pumping rate $P$ ) as also indicated by $\tau \equiv \tau_{\mathrm{c}}(P+\gamma) / P$ from eq 4. As illustrated in Figure 4 we obtained best fit to their data for $\sigma=10$ and $W_{12}=0.095 \mathrm{~ms}^{-1}$. 


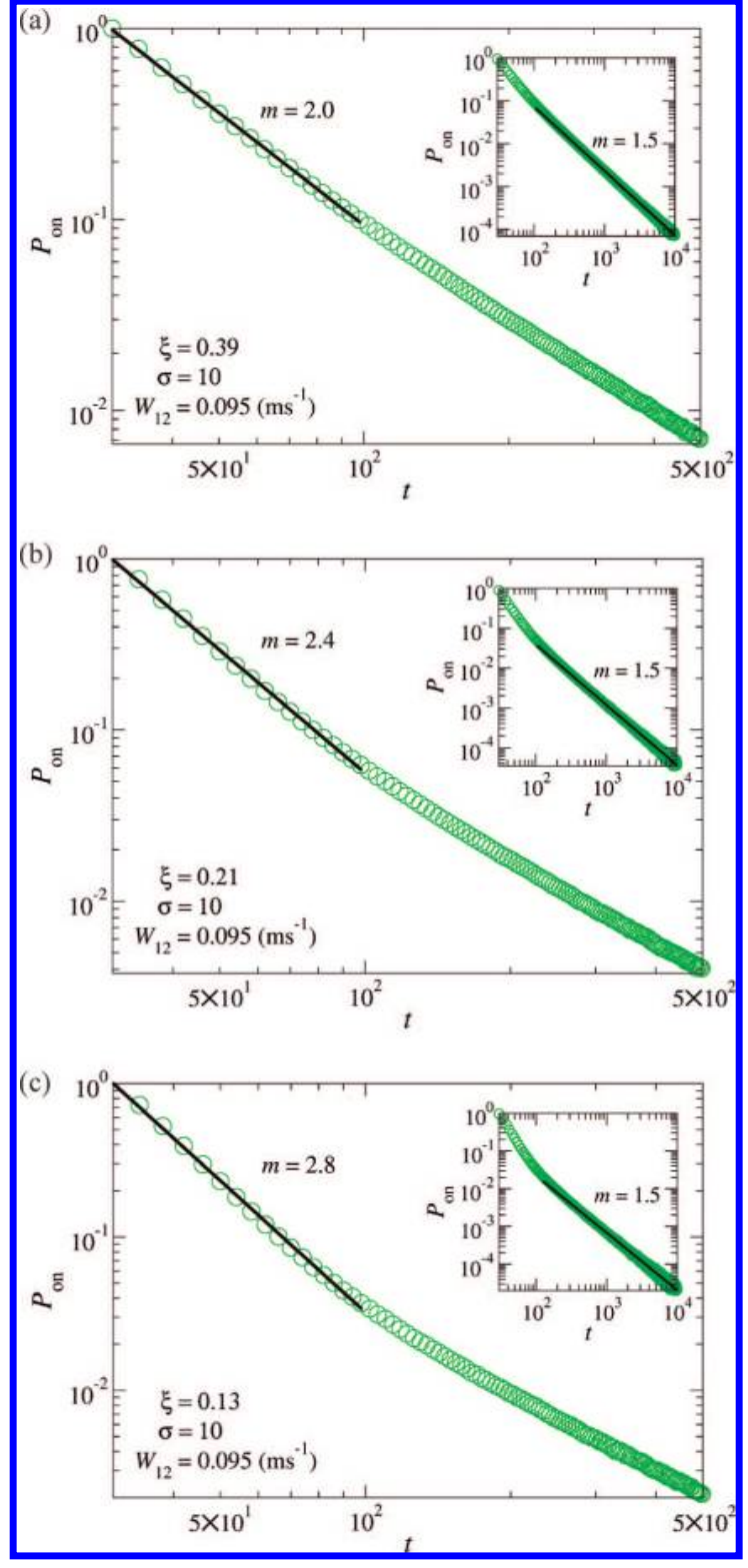

Figure 4. $\log -\log$ plot of $P_{\text {on }}(t)$ for (a) $\xi=0.39$, (b) $\xi=0.21$, and (c) $\xi=0.13$. In all cases, $W_{12}=0.095 \mathrm{~ms}^{-1}, 9.5 \times 10^{7}$ events, $\sigma=$ 10 , time unit $=1 \mathrm{~ms}$, bin size $=4 \mathrm{~ms}$ were used. The main figures illustrate the inverse power law behavior in short time with a corresponding exponent $m$ changing from 2.0, 2.4, and then to 2.8 when $\xi$ decreases 2 and 4 times. All curves have a long-time exponent of 1.5 as shown in the insets. We have systematically varied the parameter settings for $\tau, \xi$, and $W_{12}$ to obtain the desired exponent value according to Yeow et al., ${ }^{16}$ where $m$ was observed to change from 2.8 to 2.4 and then to 2.0 when the light intensity is doubled and quadrupled.

\section{Conclusions}

In conclusion, in this study of single-chromophore fluorescence intermittency we present a mechanism to explain why the exponent of the inverse power law far exceeds 2 and decreases at a higher light intensity as observed experimentally by Yeow et al. ${ }^{16}$ This unusual behavior has never been observed in semiconductor quantum dots. Our more generalized model presented here serves as a platform for physical description of general single-particle blinking phenomena commonly observed in semiconductor nanocrystals, single organic chromophores, and even large biomolecules. The main cause for a larger exponent in the inverse power-law decay is due to a broad population sink that greatly facilitates the speedy loss of the

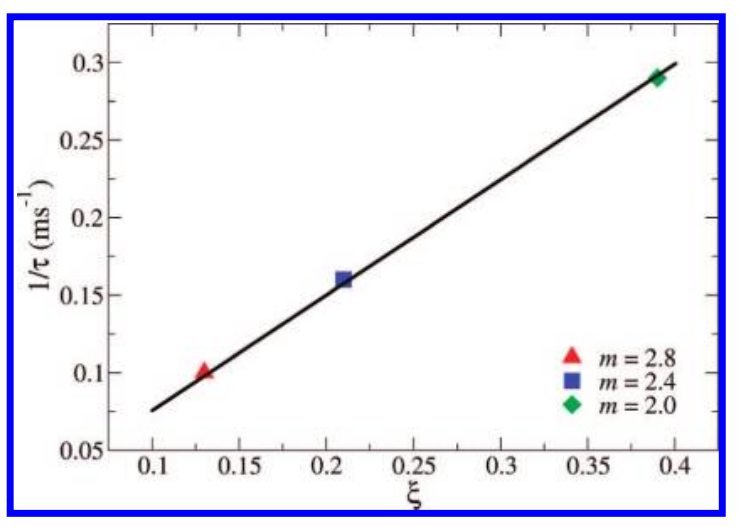

Figure 5. The simulation data (denoted as the discrete points: $m=$ $2.8,2.4$, and 2.0 with $1 / \tau$ at $0.10,0.16$, and $0.29 \mathrm{~ms}^{-1}$, respectively) for the experimental results of Yeow et al. ${ }^{16}$ at three light intensities. Consistent with the photoinduced diffusion mechanism, these data points lie in an ideal line when the light intensity and the diffusion correlation time were increased simultaneously by a factor of 2 and 4 .

light state population. In the limit of a very narrow sink such as a Dirac $\delta$-function in the previous model, the exponent could never exceed 2.

In the single-chromophore experimental data of Yeow et al., ${ }^{16}$ the power-law decay with an exponent greater than 2 only covers about one decade. As illustrated in Figures 3 and 4 from our simulations, the short-time decay is nonexponential, and one cannot characterize the decay by a simple power law with one exponent for the entire time range covering both very short time and very long time regimes. Here we used an effective exponent to characterize such a fast, short-time decay for the reported experimental observation time range. As the bin time gets even shorter, we would expect an even greater exponent at a much shorter time, although the dynamics is more dominated by the 1-D diffusion-controlled reaction regime and the long-time blinking statistics should follow the usual inverse power-law decay with an exponent of 1.5 for normal diffusion or an exponent between 2 and 1 for anomalous diffusion. This work shows that the short-time dynamics involving diffusion with a finite sink width, the blinking statistics in neither a single exponential decay nor simple inverse power-law decay. We chose to characterize the short-time decay by an effective powerlaw exponent because it would be easier to compare with the usual long-time power-law decays commonly observed in these single-particle systems as well as to directly compare with the curves reported by Yeow et al. ${ }^{16}$ who also used an inverse power law to fit their data. We think such a characterization might be better than using a multiexponential fit to describe the shorttime behavior but suddenly switching to an inverse power law for the longer time behavior. Using a multiexponential fit, it requires more fitting parameters such as a decay time constant and a pre-exponential factor for each component, whereas using power-law description it requires the exponent and the proportional constant.

According to Empedocles and Bawendi, ${ }^{18}$ the light state involves spectral diffusion, exhibiting stochastic changes of the emission peak, and such diffusion can be influenced by light. As shown in eq 4, the effective diffusion correlation time is light-intensity dependent, and diffusion becomes faster at a higher light intensity. At a higher intensity with faster diffusion, because a shorter time is spent within the Gaussian-shaped sink area, the population diffuses more effectively away from the sink. Therefore, the population decay becomes slower and the exponent for the inverse power law becomes smaller. Such a light-intensity effect on the exponent is consistent with the 
experimental observation by Yeow et al. ${ }^{16}$ According to this model, even though the initial slope for the inverse power law for the chromophores can be much greater than 2 , we expect it to decrease to 1.5 at a longer time and finally becomes an exponential decay at a much longer time where the DCET model ${ }^{14}$ becomes valid. For anomalous diffusion with a Dirac $\delta$-function sink, the exponent is different from 1.5 but could not exceed 2. The case of a broad sink involving anomalous diffusion is much more difficult to simulate and will be investigated in the future. It will be of great interest to test our model predictions more systematically using the same chromophores or other systems.

Acknowledgment. J. Tang thanks the support of the Academia Sinica and National Science Council of Taiwan under the program No. 96-2113-M-001-032-MY3, and D. H. Lee acknowledges the support during his teaching tenure at the Guang Hua Junior High School, Hsinchu.

\section{References and Notes}

(1) Nirmal, M.; Dabbousi, B. O.; Bawendi, M. G.; Mackin, J. J.; Trautman, J. K.; Harris, T. D.; Brus, L. E. Nature 1996, 383, 802.

(2) Quantum Dots: A Doorway to Nanoscale Physics; Heiss, W. D., Eds.; Springer: Berlin, 2005.

(3) Nanoparticles: Building Blocks for Nanotechnology; Rotello, V., Ed.; Kluwer Academic: New York, 2004.
(4) (a) Shimizu, K. T.; Woo, W. K.; Fisher, B. R.; Eisler, H. J.; Bawendi, M. G. Phvs. Rev. Lett. 2002, 89, 117401. (b) Chung, I.; Bawendi, M. G. Phys. Rev. B 2004, 70, 165304.

(5) (a) Kuno, M.; Fromm, D. P.; Hamann, H. F.; Gallagher, A.; Nesbitt, D. J. J. Chem. Phys. 2000, 112, 3117. (b) Kuno, M.; Fromm, D. P.; Johnson, S. T.; Gallagher, A.; Nesbitt, D. J. Phvs. Rev. B 2003, 67, 125304.

(6) Verberk, R.; Van Oijen, A. M.; Orrit, M. Phvs. Rev. B 2002, 66, 233202.

(7) (a) Cichos, F.; Martin, J.; Von Borczyskowski, C. Phys. Rev. B 2004, 70, 115314. (b) Cichos, F.; von Borczyskowski, C.; Orrit, M. Curr. Opin. Colloid Interface Sci. 2007, 12, 272.

(8) Biju, V.; Makita, Y.; Nagase, T.; Yamaoka, Y.; Yokoyama, H.; Baba, Y.; Ishikawa, M. J. Phys. Chem. B 2005, 109, 14350.

(9) Wang, S.; Querner, C.; Drndic, M.; Emmons, T.; Crouch, C. H. $\underline{J}$. Phvs. Chem. B 2006, 110, 23221.

(10) Pelton, M.; Smith, G.; Scherer, N. F.; Marcus, R. A. Proc. Natl. Acad. Sci. U.S.A. 2007, 104, 14249.

(11) (a) Wang, J.; Wolynes, P. Phys. Rev. Lett. 1995, 74, 4317. (b) Lee, C. L.; Lin, C. T.; Stell, G.; Wang, J. Phvs. Rev. E 2003, 67, 41905. (c) Zhou, Y.; Zhang, Q.; Stell, G.; Wang, J. J. Am. Chem. Soc. 2003, 125, 6300 .

(12) Lee, C. L.; Stell, G.; Wang, J. J. Chem. Phvs. 2003, 118, 959.

(13) Margolin, G.; Barkai, E. J. Chem. Phvs. 2004, 121, 1566.

(14) (a) Tang, J.; Marcus, R. A. Phys. Rev. Lett. 2005, 95, 107401. (b) Tang, J.; Marcus, R. A. J. Chem. Phvs. 2005, 123, 054704. (c) Tang, J.; Marcus, R. A. J. Chem. Phvs. 2005, 123, 204511.

(15) Tang, J. J. Chem. Phvs. 2007, 127, 111105.

(16) Yeow, E. K. L.; Melnikov, S. M.; Bell, T. D. M.; De Schryver,

F. C.; Hofkens, J. J. Phvs. Chem. A 2006, 110, 1726.

(17) Sumi, H.; Marcus, R. A. J. Chem. Phvs. 1986, 84, 4894.

(18) Empedocles, S. A.; Bawendi, M. G. Science 1997, 278, 211.

JP801810P 